\section{Tuberculous Tenosynovitis and Ulnar Bursitis of the Wrist}

\author{
Sang-Min Lee, MD, Won-Jae Lee, MD, Ah-Rom Song, MD
}

Department of Physical Medicine \& Rehabilitation, Veterans Health Service Medical Center Medical Center, Seoul, Korea

Tuberculous infection of the hand is a rare form of extrapulmonary tuberculosis that, left untreated, can cause serious joint and tendon damage. We report the case of a 65 -year-old male patient who presented with an insidious flexor weakness of the fifth finger but without any history of trauma. Magnetic resonance imaging revealed a partial tear of the fifth finger flexor tendon with a fluid collection in the tendon sheath and in the flexor compartment along the ulnar side. Under ultrasound guidance, this fluid collection was aspirated and analyzed for Mycobacterium tuberculosis using polymerase chain reaction. The assay was positive for the microorganism, confirming the diagnosis of tuberculous tenosynovitis and ulnar bursitis of the wrist. The early suspicion and diagnosis of this extrapulmonary disease facilitated anti-tuberculous chemotherapy and helped avoid a hasty injection of corticosteroid.

Keywords Tenosynovitis, Tuberculosis, Polymerase chain reaction

\section{INTRODUCTION}

Tuberculous infection of the musculoskeletal system accounts for $10 \%$ of all extrapulmonary cases of tuberculosis. In musculoskeletal tuberculous infections, the involvement of tuberculosis in peripheral joints and tendons is rare [1]. If left untreated, tuberculosis can cause serious joint and tendon damage as well as the spread of mycobacteria into the surrounding bursa, muscle, and other soft tissues. Concurrent active pulmonary tuberculosis are present in less than $50 \%$ of the patients [2].

Received June 29, 2012; Accepted September 13, 2012

Corresponding author: Won-Jae Lee

Department of Rehabilitation Medicine, VHS Medical Center, 53 Jinhwangdo-ro 61-gil, Gangdong-gu, Seoul 134-791, Korea

Tel: +82-2-2225-1479, Fax: +82-2-2225-1579, E-mail: braddom@nate.com

(c) This is an open-access article distributed under the terms of the Creative Commons Attribution Non-Commercial License (http://creativecommons. org/licenses/by-nc/3.0) which permits unrestricted noncommercial use, distribution, and reproduction in any medium, provided the original work is properly cited.

Copyright $\odot 2013$ by Korean Academy of Rehabilitation Medicine
There have been a few reports on tuberculous tenosynovitis in the Korean population [3,4]. All of these previous cases were diagnosed from tissue obtained during operative synovectomy. However, to the best of our knowledge, there has been no report on tuberculous tenosynovitis which was atypically confined to the fifth finger flexor and ulnar bursa of the wrist and was diagnosed by bursal fluid aspiration without surgical intervention. We report herein an extremely rare case of tuberculous tenosynovitis of the fifth finger flexor associated with ulnar bursitis of that wrist, which was diagnosed by using polymerase chain reaction (PCR).

\section{CASE REPORT}

A 65-year-old man presented with a 1-week history of an insidious flexor weakness of the right fifth finger. All traumatic causes were ruled out. Physical examination revealed paresthesia over the fifth finger and hypothe- 

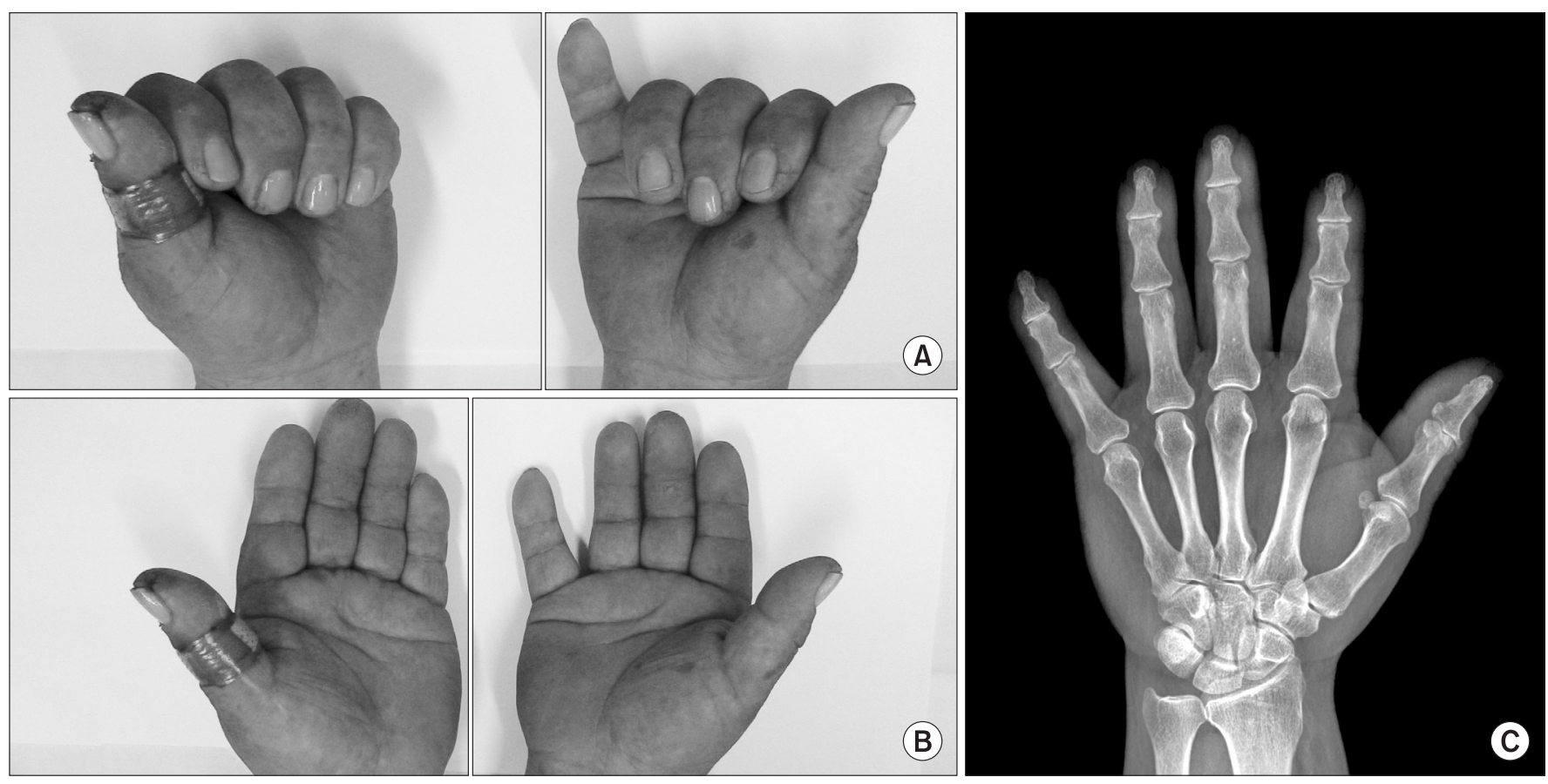

Fig. 1. Photographs of the patient demonstrating weak flexion (A) and adduction (B) of the right fifth finger. In the hand radiograph $(\mathrm{C})$, no abnormal findings in bone, joint, and soft tissue are identified.

nar region of the right hand. According to the Medical Research Council scale [5], the strength of the right fifth finger flexor was grade 2 , while the strengths of the fifth finger adductor and fourth finger flexor were both grade 3 (Fig. 1A, 1B). The muscle strengths of the right upper extremity, including the first dorsal interosseous, abductor digiti minimi and flexor carpi ulnaris muscles, was all grade 5 . No significant atrophy was identified on the right hand and forearm. Simple X-rays revealed no abnormal findings in bone, joint, and soft tissue (Fig. 1C).

The patient had been treated for diabetes mellitus and hypertension over a period of 5 years at an outpatient clinic. A chest radiograph revealed tiny calcific nodules, suspicious for old tuberculous infection; however, no significant differences were found in patterns and sizes of these lesions when compared to previous films. There was no past or family history of tuberculosis. He did not have any complaints of respiratory symptoms, including cough, sputum, or chest discomfort. The levels of white blood cell count, erythrocyte sedimentation rate, and C-reactive protein were within normal range. However, plasma glucose level and glycosylated hemoglobin level were $477 \mathrm{mg} / \mathrm{dL}$ and $13.1 \%$, respectively, which revealed poorly controlled diabetes mellitus.

Electrodiagnostic studies were performed in the right upper extremity to rule out an atypical type of ulnar neuropathy. Nerve conduction studies of latency, amplitude, and conduction velocity exhibited normal findings in motor and sensory components. Abnormal needle electromyographic findings, including abnormal spontaneous activities and motor unit action potentials, were not observed.

For further evaluation, contrast-enhanced magnetic resonance imaging (MRI) of the right hand was performed, and this revealed a partial tear of the fifth finger flexor tendon with a fluid collection in the tendon sheath and in the flexor compartment along the ulnar side. This finding was suggestive of flexor tenosynovitis associated with ulnar bursitis (Fig. 2). There were no abnormal findings in bone, soft tissue, or neurovascular structure.

Based on the spontaneous occurrence of flexor tenosynovitis along the ulnar side and tiny calcific nodules in chest radiographs, a decision was made for a PCR analysis to identify the potential infectious cause. Under ultrasound guidance, an 18-gauge needle was introduced into the hypoechoic area beneath the interrupted fifth flexor tendon (Fig. 3A). From that point, $0.3 \mathrm{~mL}$ of synovial fluid, which was slightly turbid and yellowish, was aspirated. From the area, but did not contain loose bodies. Nested PCR analysis was positive for Mycobacterium 

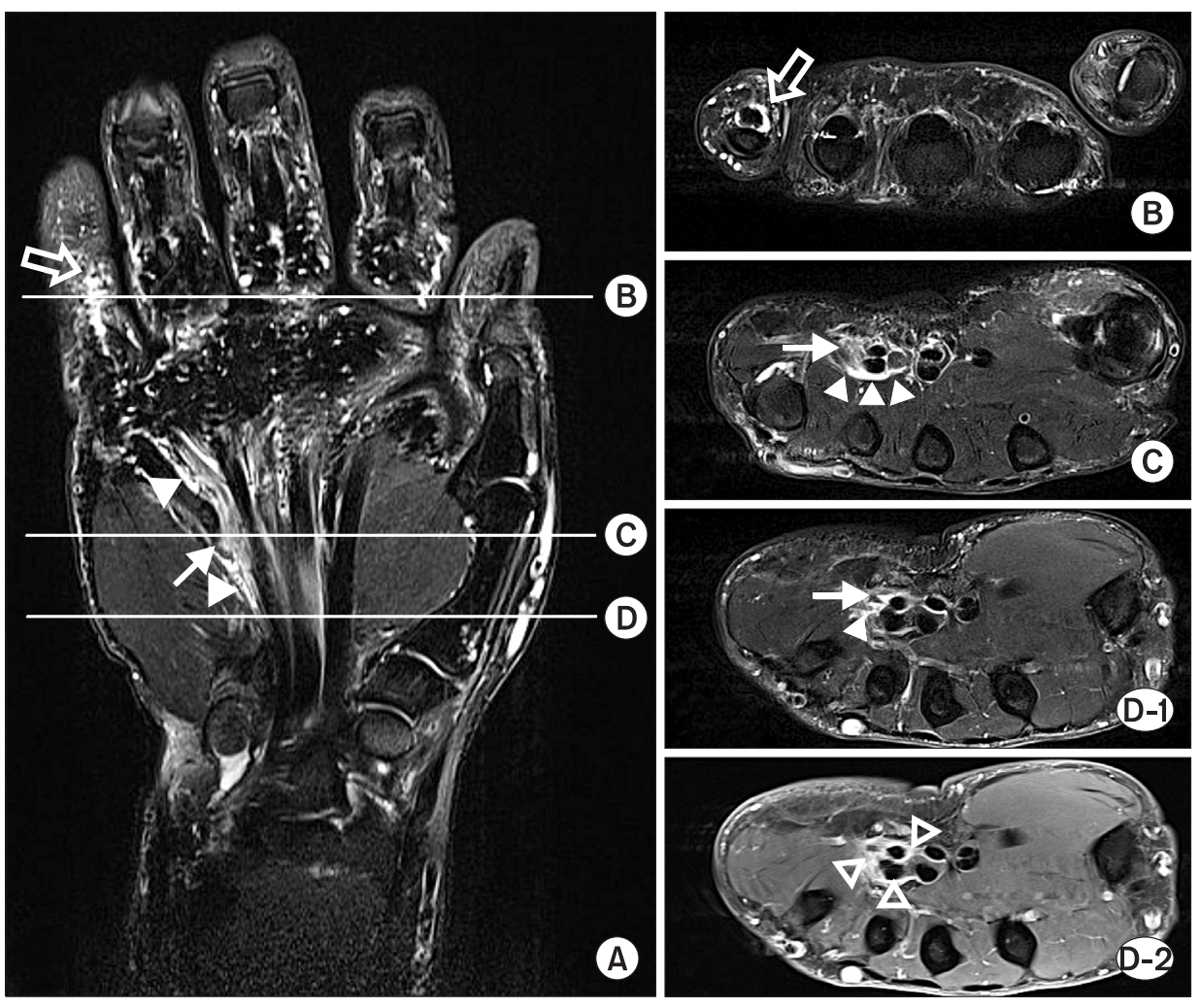

Fig. 2. On fat-suppressed T2weighted image of the right hand, the coronal view (A) shows a partial tear of the fifth finger flexor tendon (arrow), a fluid collection

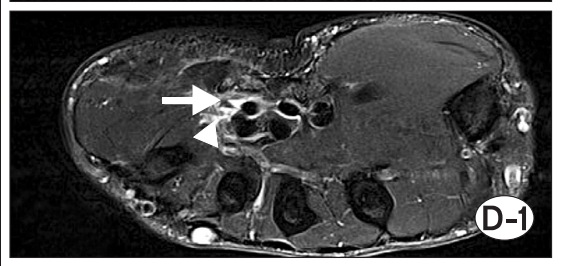
in the tendon sheath (open arrow), contiguous with the flexor compartment along the ulnar side (arrowheads). The axial views were obtained at the levels of the proximal phalanx (B), mid-palm

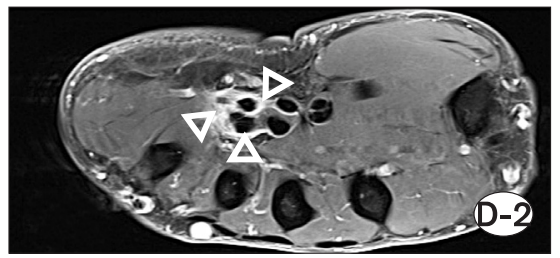
(C) and proximal palm (D-1). The contrast-enhanced T1-weighted axial image of the proximal palm (D-2) shows a thickened synovium of the ulnar bursa (open arrowheads).
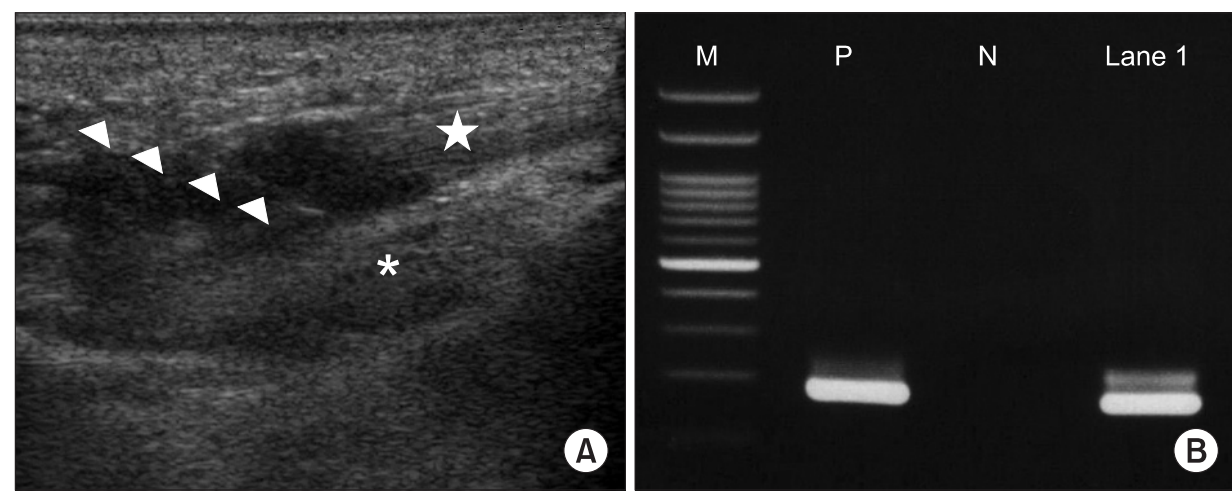

Fig. 3. Ultrasound-guided fluid aspiration (A) was conducted using an 18-gauge needle (arrowheads) at the point of the ulnar bursa between the fifth flexor digitorum (star) and the palmar interosseous muscle (asterisk). Nested polymerase chain reaction analysis (B) of the fluid revealed the presence of Mycobacterium tuberculosis. M, DNA molecular weight marker; P, positive control; N, negative control; Lane 1, patient sample.

tuberculosis gene probe in the aspirated fluid, confirming the diagnosis of tuberculous tenosynovitis (Fig. 3B). A follow up serologic test was negative for human immunodeficiency virus (HIV) antibody.

Although partial tear of fifth finger flexor tendon was highly suspected based on the MRI finding and motor weakness, a nonoperative medical treatment was preferentially followed due to the uncontrolled diabetes mellitus.
The patient was started on combination chemotherapy of isoniazid, rifampin, pyrazinamide, and ethambutol along with additional hypoglycemic agents. Two weeks after the commencement of therapy, paresthesia along the ulnar side had begun to improve; however, there were no interval changes to the motor weakness of the fifth finger flexor. Extension block splint and range of motion exercise were maintained to support fourth and fifth finger flexor ten- 
dons. Three months later, sensory symptom disappeared and motor weakness had improved to grade 4 .

\section{DISCUSSION}

Since 1950s, the incidence of tuberculous infection has gradually decreased due to anti-tuberculous chemotherapy. However, this trend has been reversing recently because of the increased number of patients infected with HIV, bacterial resistance to medications, and increased international travel and immigration [6]. Thus, the incidence of extrapulmonary tuberculosis, including musculoskeletal tuberculosis, has also been rising [2]. The present case of tuberculous tenosynovitis of the hand highlights the need for increased awareness of musculoskeletal tuberculosis to clinicians. Such unusual presentation of decreased strength and paresthesia in the fifth finger and hypothenar region, associated with finger flexor tenosynovitis and ulnar bursitis in MRI findings, should draw more suspicion for tuberculous tenosynovitis of the hand.

Tuberculous tenosynovitis most commonly occurs in the hand. For reasons unknown, the flexor side is more commonly involved than the extensor side, and the ulnar border is more commonly involved than the radial border. Swelling with mild pain and limitation of movement can be observed; however, tuberculous tenosynovitis has a gradual onset and progresses slowly. The insidious nature of this presentation is a cause for notable delay in establishing the proper diagnosis, and this may lead to tendon rupture [2]. Because of the unusual onset and clinical presentation, we should carefully perform history taking and physical examination, to differentiate tuberculous tenosynovitis from other diseases.

Anatomically, the flexor synovial sheath of the fifth finger is usually continuous with the ulnar bursa of the common flexor tendon compartment [7]. For this reason, tuberculous tenosynovitis is frequently accompanied by tuberculous bursitis [8]. The characteristic MRI finding of tuberculous tenosynovitis is a prominent synovial thickening, combined with fluid collection in the tendon sheath [1]. In the case of our 65-year-old patient, the MRI study revealed a fluid collection in the ulnar bursa and the fifth finger flexor digitorum tendon sheath. In addition to the patterns of fluid collection, the partial tear in the tendon of the fifth finger flexor and synovial thicken- ing of flexor compartment around the wrist were suggestive of infectious flexor tenosynovitis, especially of $\mathrm{Myco}$ bacterium tuberculosis.

Ultrasound-guided fluid aspiration helps to differentiate the causes of tenosynovitis [9]. Infectious causes must be differentiated from non-infectious etiology because treatment outcomes and prognoses are vastly different. For example, corticosteroid injections-a conservative treatment modality in non-infectious bursitis-can have a disastrous consequence in an infectious bursitis. In this case, the positive result of PCR for Mycobacterium tuberculosis was confirmed from the ultrasound-guided aspiration. The PCR assay, a molecular biologic technique using nucleic acid amplification, is a highly sensitive method for detecting Mycobacterium tuberculosis and requires only a few days to obtain confirmation [10]. In contrast, the sensitivity of conventional acid-fast bacillus staining is low and inappropriate for confirmation. Mycobacterium tuberculosis cultures require a long incubation time ( 6 or more weeks), and is not as useful clinically as it once was because of the delay it would cause in treatment. In conclusion, the possibility of tuberculous tenosynovitis should be considered for an insidious hand weakness that occurs in the absence of external injuries. Characteristic MRI findings and the anatomic correlation of flexor tendon sheath with wrist bursa are helpful in differentiating tuberculous tenosynovitis from other causes. Using ultrasound-guided aspiration and PCR, we could confirm the diagnosis of tuberculous tenosynovitis non-invasively and rapidly, as compared to an open surgical biopsy. The early diagnosis facilitated proper management in the case of tuberculous tenosynovitis in this patient and helped to avoid a hasty injection of corticosteroids.

\section{CONFLICT OF INTEREST}

No potential conflict of interest relevant to this article was reported.

\section{REFERENCES}

1. Hsu CY, Lu HC, Shih TT. Tuberculous infection of the wrist: MRI features. AJR Am J Roentgenol 2004;183: 623-8.

2. Pattamapaspong N, Muttarak M, Sivasomboon C. Tu- 
berculosis arthritis and tenosynovitis. Semin Musculoskelet Radiol 2011;15:459-69.

3. Chung PH, Kang S, Kim JP, Lee SH. Carpal tunnel syndrome secondary to tuberculous tenosynovitis: a case report. J Korean Orthop Assoc 2002;37:806-9.

4. Min HJ, Chung YK. Tuberculous tenosynovitis of flexor tendon of the hand. J Korean Soc Plast Reconstr Surg 2010;37:708-11.

5. Florence JM, Pandya S, King WM, Robison JD, Baty J, Miller JP, et al. Intrarater reliability of manual muscle test (Medical Research Council scale) grades in Duchenne's muscular dystrophy. Phys Ther 1992;72:115-22.

6. Rieder HL. The global importance of tuberculosis. Ther Umsch 2011;68:359-64.
7. Moore KL, Dalley AF. Clinically oriented anatomy. 4th ed. Philadelphia: Lippincott Williams \& Wilkins; 1999.

8. Jaovisidha S, Chen C, Ryu KN, Siriwongpairat P, Pekanan P, Sartoris DJ, et al. Tuberculous tenosynovitis and bursitis: imaging findings in 21 cases. Radiology 1996;201:507-13.

9. Chau CL, Griffith JF. Musculoskeletal infections: ultrasound appearances. Clin Radiol 2005;60:149-59.

10. Michos AG, Daikos GL, Tzanetou K, Theodoridou M, Moschovi M, Nicolaidou P, et al. Detection of Mycobacterium tuberculosis DNA in respiratory and nonrespiratory specimens by the Amplicor MTB PCR. Diagn Microbiol Infect Dis 2006;54:121-6. 